\title{
Non-linear Mapping for Feature Extraction
}

\author{
P. Scheunders ${ }^{1}$, S. De Backer ${ }^{1}$ and A. Naud ${ }^{2}$ \\ 1 Vision Lab, Department of Physics, University of Antwerp, Groenenborgerlaan 171, \\ 2020 Antwerpen, Belgium, \\ 2 Department of Computer Methods, University Nicolai Copernici, \\ ul. Grudziadzka 5, 87-100 Toruń, Poland
}

\begin{abstract}
Mapping techniques have been regularly used for visualization of high-dimensional data sets. In this paper, mapping to $d \geq 2$ is studied, with the purpose of feature extraction. Two different nonlinear techniques are studied: self-organizing maps and auto-associative feedforward networks. The non-linear techniques are compared to linear Principal Component Analysis (PCA). A comparison with respect to feature extraction is made by evaluating the reduced feature sets ability to perform classification tasks. The experiments involve an artificial data set and grey-level and color texture data sets.
\end{abstract}

\section{Introduction}

In many classification problems large feature vectors are generated to be able to describe complex objects and to distinguish between them. On the other hand, the amount of available data points is limited in many practical situations. For a classifier, the estimation of the class probability distributions in these sparsely sampled high-dimensional data spaces is troublesome, and generally the liability of the obtained classification results is affected.

To avoid these problems, the dimension of the feature space is reduced. This can be done in several ways. The easiest way is to select a limited set of features out of the total set. The classification performance serves as a measure for the selection process. Some well-known feature selection techniques are forward selection and branche and bound techniques [1]. Another way is feature extraction. Here, features are extracted as functions (linear or non-linear) of the original set of features. A well known linear feature extraction technique is Principal Component Analysis (PCA), which rotates the original feature space, before projecting the feature vectors onto a limited amount of axes.

Some well known non-linear techniques are Multidimensional Scaling [2] and Sammon's mapping [3]. With the development of neural networks, new possibilities for non-linear mapping were created. Amongst them, Self-Organizing Maps are probably the most well-known [4]. Other ways include auto-associative feedforward networks [5]. Although the mentioned techniques are theoretically capable of performing a non-linear mapping into a space with arbitrary dimension, most applications report on mappings to $d=2$, with the purpose of visualizing the data (some recent applications are found in $[6,7]$ ). 
In this paper, a study is performed on non-linear dimensionality reduction to $d \geq 2$. Two techniques are evaluated: Kohonen's self-organizing map and an auto-associative feedforward neural network. The performance of these techniques is evaluated and compared to linear mapping (PCA). This is done by examening the mapped feature space's ability to perform classification tasks. Apart from an artificial data set, real world applications are studied in the field of texture analysis where high-dimensional wavelet-based feature sets from greylevel and color texture images are used. We will demonstrate that the non-linear feature extraction techniques lead to feature sets which improve classification performance compared to linear mappings.

\section{Non-linear Mapping}

Let us start with $N$ pattern vectors $\boldsymbol{y}_{i}$ in a $D$ dimensional feature space. We want to build a mapping that projects each of them to a $d$ dimensional feature space $(d<D)$, obtaining low dimensional pattern vectors $\boldsymbol{x}_{i}$. Building this map will correspond to minimizing some error function $E\left(\boldsymbol{y}_{i}\right)$.

\subsection{Self-organizing Map (SOM)}

SOM was proposed by Kohonen $[8,4]$ to build topology preserving mappings. A set of neurons is defined, which are regularly positioned in a d-dimensional lattice ( $=$ the output space). The position of a neuron $k$ in the input space is given by its weight vector $\boldsymbol{W}_{k}$. During the training phase, the values of $\boldsymbol{W}_{k}$, being chosen at random initially, are altered, as to minimize the following error function:

$$
\begin{gathered}
E_{S O M}=\sum_{k} \sum_{i \in S_{k}}\left(\boldsymbol{W}_{k}-\boldsymbol{y}_{i}\right)^{2} \\
S_{k}=\left\{i ; k=\arg \min _{j}\left(\left(\boldsymbol{W}_{j}-\boldsymbol{y}_{i}\right)^{2}\right)\right\}
\end{gathered}
$$

i.e. $S_{k}$ being the set of data points which have neuron $k$ as closest neuron. After training, each data point $\boldsymbol{y}_{i}$ in the input space is assigned to one of these neurons, namely the one whose weight vector is closest to the point. Its position vector $\boldsymbol{x}_{i}$ in the output space is given by the position vector of its closest neuron in the map.

Minimization of $E$ is now performed with respect to the weight vectors $\boldsymbol{W}_{k}$. The gradient descent approach leads to the following updating rule:

$$
\boldsymbol{W}_{k}^{(t+1)}=\boldsymbol{W}_{k}^{(t)}-\alpha^{(t)} \cdot\left(\nabla E^{(t)}\right)_{k}
$$

With $\alpha^{(t)}$ the learning step, which decreases in function of $t$. This algorithm clusters the input space, but is not useful for mapping since the learning rule 
does not depend on the output space. For this reason the learning rate is replaced by a neighbourhood function $h_{c}$ which explicitely depends on the mapped space:

$$
h_{c}^{(t)}= \begin{cases}\alpha^{(t)} & \text { if } k \in N_{c}^{(t)} \\ 0 & \text { if } k \notin N_{c}^{(t)}\end{cases}
$$

where $N_{c}^{(t)}$ is the set of all neurons within a certain range of the winning neuron $c$ (i.e., the nearest element to the presented data point $\boldsymbol{y}_{i}$ ) in the output space. During training this range and $\alpha^{(t)}$ are decreased monotonically. Since neighbouring neurons in the output space will be neighbours in the input space, the mapping preserves topology.

For a fixed sampling of the output space, the number of neurons and therefor the time complexity of SOM grows exponentially. This becomes impractical when $d>4$. Therefore the following approximative strategy is applied. The total feature set is subdivided into $d / 2$ smaller sets. Each set forms a $\frac{2 D}{d}$ dimensional feature space, which is then reduced to a two-dimensional space using SOM. The total map is then obtained by combining the $d / 22$-dimensional reduced spaces.

\subsection{Auto-Associative Feedforward Neural Networks (AFN)}

In an AFN the goal is to reproduce the feature space at the output layer while obtaining a reduced representation at the hidden layer $[5,9]$. The error function is given by:

$$
E_{A F N}=\sum_{i}\left(\boldsymbol{y}_{i}-g\left(f\left(\boldsymbol{y}_{i}\right)\right)\right)^{2} .
$$

where $f$ and $g$ are the outputs of layer 2 and 3 respectively and are generally nonlinear functions. Since our aim is dimensionality reduction, the hidden layer will be representing the mapped space, the number of hidden neurons being $d$, and $f\left(\boldsymbol{y}_{i}\right)=\boldsymbol{x}_{i}$, being the mapping. For obtaining good non-linear representations, a 5-layer network is constructed, 2 layers to generate $f$, and 2 for $g$.

The gradient descent learning rule to minimize $E_{A F N}$ is given by:

$$
\boldsymbol{W}^{(t+1)}=\boldsymbol{W}^{(t)}-\alpha^{(t)} \nabla E_{A F N}^{(t)}
$$

where $\boldsymbol{W}$ is the vector of the weights of all neurons. This rule can be updated by using the standard backpropagation procedure.

Experimentally, (5) is found to be very sensitive to the value of $\alpha$.

Therefore instead of standard backpropagation, a quasi-Newton technique is used:

$$
\boldsymbol{W}^{(t+1)}=\boldsymbol{W}^{(t)}-\alpha^{(t)}\left(H_{E_{A F N}}^{(t)}\right)^{-1} \nabla E_{A F N}^{(t)}
$$

The gradient of the error is still calculated by backpropagation. At each iteration the inverse Hessian $\left(H_{E_{A F_{N}}}^{(t)}\right)^{-1}$ is estimated by the limited memory version of the Broyden-Fletcher-Goldfarb-Shanno (BFGS) procedure [10]. There, the learning rate $\alpha^{(t)}$ is updated to satisfy some optimality constraints. 


\section{Experiments and Discussion}

In the previous section, the two techniques were described as minimizations of an error function. Both error functions aim at preserving topology of the original space into the projected space. Instead of trying to analyse the quality of the mappings directly from the form of the error function, we chose to use the maps for a specific task, and compare the behaviour of the mappings by the performance for that task. Since feature extraction is an important motivation for this study, the performance of the mapping techniques will be measured by the classification performance of the obtained reduced feature set.

The experiments involve classification, in which a $D$-dimensional data space is subdivided into a given number of classes, given a training set of labeled feature vectors. The original feature space is first mapped onto a $d$-dimensional space $(d<D)$, after which classification is performed on the projected space. The classifier is a $k$-NN classifier $(k=5)$, applied together with the leave-one-out technique. The classification performances for different values of $d$ are compared.

An important property of a data set is its intrinsic dimensionality. We define the intrinsic dimensionality as the minimum number of features needed to obtain an optimal classification performance. Of course this number will depend on the feature extraction technique used. We have estimated the intrinsic dimensionality of a data set in two independent ways. First a suboptimal feature selection technique is used [11]. Here, an optimal set of features is selected from the complete set, where the classification performance of the set is the optimality criterion. Secondly, the fractal dimension of the complete data set is calculated [12].

The following three data sets are used:

1. An artificial data set, generated by defining $D+1 D$-dimensional data points in a simplex configuration (all interpoint distances are equal to 1). We have chosen $D=10$. Around these points, 10-dimensional multinormal distributions are generated. For each distribution, 100 points are generated $(N=1100)$. Each distribution forms a class (total of 11 classes). The variance of the distributions is chosen so that the Bayes error-rate (i.e. the inter-class overlap) is about $20 \%$. For this data set all features are equally important. The intrinsic dimensionality of this data set is 10 by construction.

2. A grey-level texture data base of $N=245$ images containing 5 different Brodatz textures (5 classes). A continuous wavelet transform is performed and rotation-invariant features are extracted [13]. A 39-dimensional data space is obtained. The intrinsic dimensionality of this data set is estimated to be about 3 .

3. A colour texture data base of $N=1024$ images containing 16 different textures (16 classes). The feature vectors are generated by employing a discrete wavelet transform on the $\mathrm{R}, \mathrm{G}$ and $\mathrm{B}$ plane separately and calculating the energy features of the detail images [14]. A 48-dimensional data space is obtained. The intrinsic dimensionality is estimated to be about 5 . 
In the conducted experiments, the SOM and AFN are compared to linear dimensionality reduction. For this purpose, a Principal Component Analysis ( $\mathrm{PCA})$ is performed. All the algorithms are written in $\mathrm{C}$ and implemented on $\mathrm{HP} / 9000$ series workstations.

Using SOM, the number of neurons is chosen to be 10 in each dimension (total of $10 d$ ) in the first two datasets and 50 in each dimension (total of $50 \mathrm{~d}$ ) in the third dataset. The algorithm is observed to converge after about $100 \mathrm{~N}$ iterations. Using AFN, the number of neurons is $D$ in the input and output layer, and $d$ in the middle hidden layer. In the other 2 hidden layers it is chosen to be $x=20$ for data set $1, x=30$ for data set 2 and $x=40$ for data set 3 . The total number of neurons then becomes $2 D+d+2 x$. Weights are defined between subsequent layers only. An extra weight is defined between all input neurons and all neurons of the middle layer and also between all neurons of the middle layer and all output neurons. The total number of weights becomes $W=2 D x+2 d x+2 D d$. The algorithm is observed to converge slowly, the total number of iterations being of the order of $1000 \mathrm{~N}$.

Figure 1(a) (resp. 1(b) and 1(c)) displays the classification performances (in percent) in function of $d$ for data set 1 (resp. 2 and 3).

From these curves we can deduct the following:

- Classification performance using PCA increases almost linearly with $d$ until the intrinsic dimensionality is obtained. From then on it saturates.

- Classification performance using the non-linear techniques starts off from a higher value at $d=1$, reaches an optimal value for $d$ smaller than the intrinsic dimensionality and saturates from then on. For the three techniques similar saturation values results are obtained. In the case of non-linear feature extraction however, the optimal results are obtained for lower values of $d$. Remark that SOM has been applied using the approximative strategy, which accounts for the somewhat lower optimal classification results. 


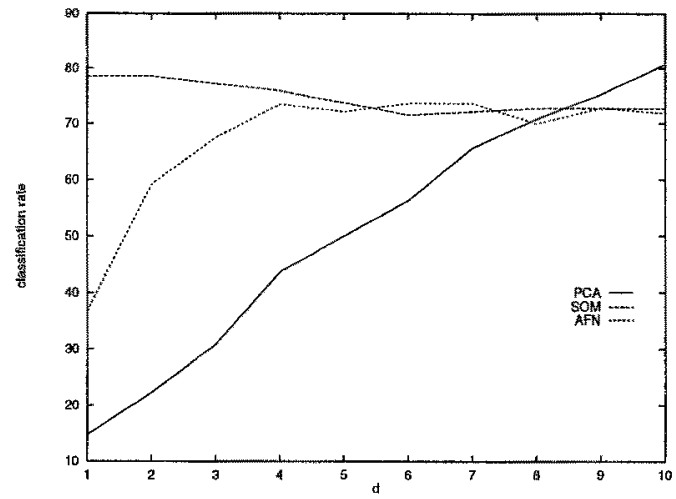

(a)

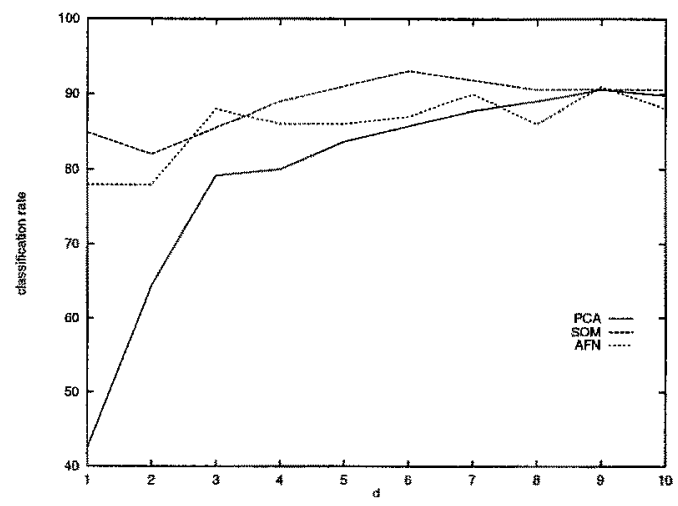

(b)

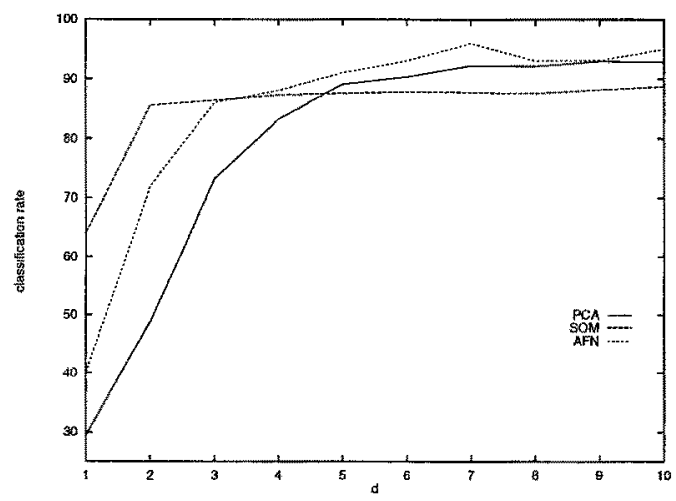

(c)

Fig. 1. Classification performance in function of $d$ (in percentage) for PCA, SOM and AFN; (a) for data set 1; (b) for data set 2 ; (c) for data set 3. 
In table 1, the classification rates are given in detail for all techniques for $d \leq 4$. Remark that this time SOM is applied without approximation. To keep the total number of neurons constant, the number of neurons in each dimension is chosen to be 2500 in $d=1,50$ in $d=2,15$ in $d=3$ and 7 in $d=4$.

\begin{tabular}{|c|c|c|c|c|}
\hline & d & Data set 1 & Data set 2 & Data set 3 \\
\hline PCA & 1 & 15 & 42 & 29 \\
\cline { 2 - 5 } & 2 & 22 & 62 & 50 \\
\cline { 2 - 5 } & 3 & 30 & 83 & 72 \\
\cline { 2 - 5 } & 4 & 43 & 89 & 83 \\
\hline SOM & 1 & 82 & 85 & 74 \\
\cline { 2 - 5 } & 2 & 80 & 88 & 88 \\
\cline { 2 - 5 } & 3 & 79 & 88 & 91 \\
\cline { 2 - 5 } & 4 & 76 & 87 & 90 \\
\hline AFN & 1 & 37 & 78 & 40 \\
\cline { 2 - 5 } & 2 & 60 & 78 & 72 \\
\cline { 2 - 5 } & 3 & 68 & 88 & 86 \\
\hline & 4 & 73 & 86 & 88 \\
\hline
\end{tabular}

Table 1. Classification performance (in percentage) of PCA, SOM and AFN for $d=1$ to 4

From this table, the superior results of SOM for very small values of $d$ becomes clear.

To give an idea about the time complexity of the techniques in real problems, the CPU-times are given in table 2 (in minutes on a HP9000/712 workstation) for the runs of table 1. No results are given for PCA, because run times were much smaller than one minute.

\begin{tabular}{|c|c|c|c|c|}
\hline & $\mathrm{d}$ & Data set 1 & Data set 2 & Data set 3 \\
\hline SOM & 1 & 17 & 13 & 67 \\
\cline { 2 - 5 } & 2 & 13 & 9 & 33 \\
\cline { 2 - 5 } & 3 & 15 & 10 & 31 \\
\cline { 2 - 5 } & 4 & 10 & 6 & 18 \\
\hline AFN & 1 & 75 & 76 & 611 \\
\cline { 2 - 5 } & 2 & 120 & 100 & 790 \\
\cline { 2 - 5 } & 3 & 135 & 120 & 840 \\
\cline { 2 - 5 } & 4 & 150 & 130 & 893 \\
\hline
\end{tabular}

Table 2. The CPU-times in minutes on a HP9000/712 workstation for the experiments of table 1.

From this table, the following conclusions can be drawn. Because the total number of neurons is constant, the run times using SOM can be kept feasible. 
AFN converges much slower ( 10 times more iterations) which leads to excessively high run times (10 to 20 times higher than SOM).

\section{Conclusion}

In this paper, a study is performed on non-linear feature extraction. Two techniques were studied: Kohonen's self-organizing map (SOM) and an auto-associative feedforward neural network (AFN). Classification experiments on the extracted feature sets reveal that the non-linear extracted features perform better than PCA based feature extraction. In particular, an optimal classification result is obtained for a smaller number of extracted features. For very small values of $d$, SOM performs superior. For higher values of $d$, SOM has to be approximated, and AFN performs somewhat better, but at the expense of higher computation times.

\section{References}

1. Devijver, P.A., Kittler, J.E.: Pattern recognition: a statitical approach. Prentice/Hall, Englewood Cliffs, New Jersey (1982) Chapter 5

2. Kruskal, J.B.: Non metric multidimensional scaling: a numerical method. Psychometrika. 29 (1964) 115-129

3. Sammon, J.W.: A nonlinear mapping for data analysis. IEEE Transactions on Computers. C-18 (1969) 401-409

4. Kohonen, T.: Self-Organizing Maps. Springer-Verlag (1995)

5. Baldi, B., Hornik, K.: Neural networks and principal component analysis: Learning from examples without local minima. Neural Networks, 2 (1989) 53-58

6. Kraaijveld, M.A., Mao, J., Jain, A.K.: A nonlinear projection method based on kohonen's topology preserving maps. IEEE Transactions on Neural Networks. 6(3) (1995) 548-559

7. Mao, J., Jain, A.K.: Artificial neural networks for feature extraction and multivariate data projection. IEEE Transactions on Neural Networks. 6(2) (1995) 296-317

8. Kohonen, T.: The self organizing map. Proc. of the IEEE. 78 (1990)

9. Kramer, M.A.: Nonlinear principal component analysis using autoassociative neural networks. AIChE Journal. 37(2) (1991) 233-243

10. Liu, D.C., Nocedal J.: On the limited memory bfgs method for large scale optimization. Mathematical Programming. 45 (1989) 503-528

11. Pudil, P., Novovicova, J., Kittler, J.: Floating search methods in feature selection. Pattern Recognition Letters. 15 (1994) 1119-1125

12. Sarkal, N., Chaudhuri, B.B.: An efficient approach to estimate fractal dimension of textural images. Pattern Recognition. 25 (1992) 1035-1041

13. Vautrot, P., Van de Wouwer, G., Scheunders, P., Livens, S., Van Dyck, D.: Continuous wavelets for rotation-invariant texture classification and segmentation. Unpublished (1997)

14. Van de Wouwer, G., Scheunders, P., Livens, S., Van Dyck, D.: Colour texture classification by wavelet energy-correlation signatures. Pattern Recognition (1997) to appear 